\title{
La transformación de la universidad y los dispositivos de cuantificación
}

\author{
University transformation and quantification devices
}

\author{
Carla FARDELLA-CISTERNAS' \\ Vicente SISTO CAMPOS ${ }^{2}$ \\ Felipe JIMÉNEZ VARGAS²
}

\begin{abstract}
Resumen
Las políticas de Educación Superior en Chile les demandan a las Universidades la instalación de dispositivos de gestión orientados a organizar, cuantificar y monitorear el trabajo académico. Pensando en las implicaciones del uso de estos dispositivos de gestión, este trabajo presenta los resultados de un análisis discursivo de 95 documentos de trabajo (Reglamentos, Bases de concurso, Formularios de acreditación) para conocer las interpelaciones que realiza a la labor universitaria. Mediante el método de análisis de discurso, se caracteriza la actuación de los documentos oficiales que regulan y transforman el trabajo académico. El estudio realizado evidencia que los dispositivos de gestión del Trabajo académico performan el trabajo mediante acciones tales como: establecer jerarquías entre las múltiples tareas de un académico y entre académicos, mediante criterios que no han sido discutidos por la comunidad profesional; objetivar procesos laborales y asumir consensos en torno a ello, desconociendo disputas y desacuerdos actuales; omitir el contexto de producción académica, construyendo una imagen del trabajo como proceso individual; y finalmente instando relaciones laborales individualizadas y competitivas.
\end{abstract}

Palabras clave: Análisis de discurso; Dispositivos de Gestión; Modernización del sector público; Rendición de cuentas; Trabajo académico.

\begin{abstract}
In Chile, higher education policies have required universities to adopt management tools related to the monitoring and quantification of academic work. Accordingly, this paper presents the results of a documentary study of 95 official documents concerning academic work (Regulations, Scholarship and Grant Application Guidelines and Accreditation Application Forms) in order to understand the regulations pertaining to academic work. Discourse analysis was used to determine how these documents are used in the university environment to regulate and transform the academic work. The present study shows that management tools adopted characterize the academic work through actions such as: establish hierarchies among the multiple tasks of a faculty member and among faculty members using criteria that have not been discussed by the academic community; objectify work processes and reach consensus over them, disregarding current disputes and disagreements; omit the context of academic production creating an image of work as an individual process; and finally urge the establishment of individualizing and competitive work relationships.
\end{abstract}

Keywords: Discourse analysis; Management devices; New public management; Accountability; Academic work.

$\because$ V V

1 Universidad Andrés Bello, Facultad de Educación, Núcleo de Educación. Av. Quillota, 980, Viña del Mar, Chile. Dirección de correspondencia/Correspondence to: C. FARDELLA-CISTERNAS. E-mail:<fardellacarla@hotmail.com>.

2 Pontificia Universidad Católica de Valparaíso, Facultad de Filosofia y Educación, Escuela de Psicología. Viña del Mar, Valparaíso, Chile.

Apoio: Comisión Nacional de Investigación Científica y Tecnológica (Proyectos Fondecyt n 3150374 y n 1151209). 
Durante las últimas décadas la Universidad ha vivido una progresiva transformación de sus formas de funcionamiento. A nivel general, vemos que las demandas estatales por el aumento de cobertura y el autofinanciamiento de la Educación superior han avanzado junto a la incorporación de lógicas empresariales del mundo privado para lograrlo. Esto ha impulsado cambios radicales en la gestión del espacio universitario, los cuales interpelan directamente a la fuerza laboral que debe llevarlos a cabo: los académicos (Beverungen, Dunne, \& Sorensen, 2008; Svensson, Spoelstra, Pedersen, \& Schreven, 2010). Tal como señala Altbach (2000) los académicos están en el corazón de esta nueva empresa y sin su desempeño, ninguna universidad puede ser exitosa en este proyecto.

Es así como en el espacio laboral universitario se han implementado múltiples regulaciones que reorganizan la labor académica: constantes rendiciones de cuenta en torno a la productividad investigativa y efectividad docente, indicadores y rankings de desempeño, indicadores de innovación, remuneraciones y bonos condicionados a la producción e investigaciones dependientes de la adjudicación de fondos de financiación concursables, entre otros (Blanch-Ribas \& Cantera, 2011; Ibarra, 2002).

Asociada a las nuevas regulaciones encontramos la incorporación de dispositivos de cuantificación del trabajo, como una herramienta transversal que apoya la mayoría de las nuevas regulaciones del trabajo académico. Ramírez (2013) señala que la universidad ha incorporado, con impresionante naturalidad, los dispositivos de cuantificación, seguimiento y auto-seguimiento de la producción en la gestión de la fuerza laboral académica. Moore y Robinson (2015) señalan que estos instrumentos permiten, a través de la medición de aspectos generales y específicos de la experiencia productiva de los trabajadores, realizar un monitoreo constante de la fuerza laboral. Según los autores, estos dispositivos tendrían un fuerte impacto en el sujeto, sus relaciones y procesos productivos.
Chile por su parte, ha sido considerado un caso emblemático en la implementación de políticas neoliberales en educación superior, incluyendo la instalación de estrategias de gestión, control y financiamiento basados en la rendición de cuentas en cuanto a productividad, cobertura y otros resultados. Ello en un contexto de creciente autofinanciación y reducción del rol del Estado a un regulador mediante incentivos (Gjerding, Wilderom, Cameron, Taylor, \& Scheunert, 2006; Moore, Kleinman, Hess, \& Frickel, 2011; Slaughter \& Leslie, 1997; Slaughter \& Rhoades, 2004). Por ello resulta importante conocer cómo se despliegan estos dispositivos en nuestra área y caracterizar su funcionamiento.

El presente artículo aborda cómo los instrumentos de gestión promovidos por las actuales políticas de educación superior instalan mandatos y prescripciones que modelan el trabajo académico. Mediante el análisis pragmático de discurso de una muestra de 95 documentos oficiales relativos a la nueva de gestión universitaria en Chile, se muestra el accionar de los dispositivos de gestión sobre el trabajo académico.

En una primera parte, el trabajo expone las transformaciones universitarias desarrolladas en las últimas décadas en Chile, centrándose particularmente en un contexto de nueva Gestión Pública (NPM, New Public Management) y Estado Basado en Evidencias (EBP, Evidence-Based Policies). En una segunda parte se presentan los resultados del análisis, con lo cual esperamos entregar herramientas para comprender los efectos que estas transformaciones están teniendo o pueden tener sobre el trabajo académico.

\section{El modelo neoliberal y las nuevas regulaciones del trabajo universitario: el caso chileno}

Desde fines de la década de los 70, la educación superior chilena experimentó importantes transformaciones acordes a un contexto nacional de progresiva neoliberalización. Expresión de esto es la 
reforma del financiamiento de la educación superior, disminuyendo el subsidio estatal para la educación superior y en paralelo, creando universidades privadas. De acuerdo con Bernasconi (2015) el sistema fue privatizado y se impuso un sistema de competencia entre las instituciones, públicas y privadas, como estrategia de sobrevivencia. Buena parte del gasto público en educación superior comienza a distribuirse sobre la base de proyectos concursables y de esta manera diferentes instituciones son forzadas a pelear por decrecientes recursos fiscales. Tales acciones dan cuenta de una transformación radical de la lógica de financiamiento de las universidades chilenas, lo cual es sostenido con el advenimiento de la democracia. De acuerdo a esto, la competencia y los incentivos funcionarían como un mecanismo promotor de la eficiencia.

Aparejado a una nueva estructura de financiamiento, la universidad debe asumir también una nueva lógica para organizarse y dar respuesta a esta inédita relación con el Estado. De acuerdo con Sousa, Nijs, y Hendriks (2010, p.1440) esta reorganización de la Universidad "descansa en asumir que mejor (y más) 'management' es indispensable para lograr y salvaguardar la contribución de las organizaciones públicas, incluyendo a las universidades, a la sociedad", "estimulándolas a que adopten prácticas modernas de gestión" (Sousa et al., 2010, p.15). De esta manera se comienza a configurar un paradigma gerencial en el modo de comprensión de la Universidad como organización.

En un nivel más general, podemos señalar que las transformaciones vividas por la universidad chilena encuentran su fundamento en dos lógicas propias de los estados neoliberales: La nueva gestión pública (NPM) y la Política Basada en Evidencias (EBP). A continuación explicaremos ambas lógicas a fin de exponer antecedentes que permitan ampliar la comprensión de las nuevas tendencias en la gestión del trabajo académico.

EI NPM se refiere a una forma de administración estatal, inspirada en formas de gestión de la empresa privada e implementada bajo el argumento de enmendar la densa y lenta burocracia, mediante modos y tecnologías, eficientes y competitivas, propias de la organización privada.

Enmarcado en estas ideas, el Estado emprende mecanismos tales como: descentralización y diversificación de las fuentes de financiamiento para la universidad (y otros servicios en general); el mercado como el principal mecanismo regulador a la vez que aseguramiento mínimo de la calidad de los servicios mediante instrumentos de acreditación. A su vez, estos mecanismos de acreditación de calidad van favoreciendo la expectativa de una cultura de accountability y eficacia para los procesos de trabajo (Blanch-Ribas \& Stecher, 2009; Fardella, Sisto, \& Jiménez, 2015).

Esto se traduce en la comprensión de las instituciones universitarias como organizaciones prestadoras de servicios de formación (profesional y avanzada), de investigación y asistencia técnica. Las cuales, para aumentar su productividad y calidad de servicio deben ser puestas a competir entre ellas, incorporando proveedores privados y transformando los sistemas de financiamiento estatal en otros de carácter competitivo, lo que estimularía a las instituciones a conectarse con las diversas demandas (del sector empresarial, de potenciales estudiantes, del gobierno y otros posibles clientes).

Es por ello que diversos autores hablan de la transformación del ethos y la labor universitaria, reorientando su acción formadora y de producción de conocimientos a aquello que pueda ser vendido a estudiantes, empresas y gobiernos (Ibarra, 2002; Sousa et al., 2010), así como a aquello que pueda ser visible en los indicadores de productividad a los cuales está hoy sometido el trabajo académico.

Nos referiremos ahora al EBP. Las políticas basadas en evidencias representan una lógica que se instala bajo la promesa de mejorar el desempeño de los Estados en la conducción de los asuntos públicos mediante el uso de información de alta calidad. La alta cualificación de la información estaría dada por fuentes tales como conocimiento especializado, investigación nacional e internacional, datos 
estadísticos, stakeholders y think tanks (Head, 2008, 2010). En coherencia con esto, para fundamentar normativas y regulaciones debe contarse con la mejor evidencia disponible (Castillo, 2015; Head, 2010), relevando la información de índole científica, el registro sistemático de acciones y el cálculo de prácticas y eventos a través de diversos manuales e índices. Siguiendo a Castillo (2015), ello no solamente comporta la formulación de una retórica que recurre a fuentes de información "confiable" para la promulgación de políticas, sino también incide en la construcción de instrumentos que permitan la producción de los datos para tomar decisiones basadas en ello (Castillo, 2015; Head, 2010).

El marco de la EBP provee una nueva dimensión para pensar el vínculo UniversidadEstado, sus consecuencias sobre la institución universitaria y la reorganización de su fuerza de trabajo. El sistema de Educación superior chileno, en consonancia con estas nuevas lógicas se orienta a perfeccionar un sistema de indicadores que deben orientar y visibilizar la acción de la universidad (Organización para la Cooperación y el Desarrollo Económicos, 2009; Salmi, 2009). Esto quiere decir un conjunto de indicadores que permiten una gestión universitaria de procesos complejos, basada en evidencia.

Acorde a esto, como dispositivos de gestión encontramos la proliferación de múltiples indicadores tales como: Relativos a la formación de pregrado están los años de acreditación de cada programa de acuerdo con el sistema nacional de acreditación; el porcentaje de retención de estudiantes en primer año; el tiempo de titulación; y la empleabilidad. En estudios de postgrado: cantidad de programas, de máster y doctorados acreditados; años de acreditación y tiempo de graduación. En investigación: proyectos adjudicados en concursos de Comisión Nacional de Investigación Científica y Tecnológica (principal organismo chileno de financiamiento de investigación científicoacadémica), montos adjudicados en proyectos indexadas en las bases Web of Science-ISI, Scopus y SciELO. Y en innovación: patentes solicitadas; proyectos de emprendimiento con financiamiento externo adjudicados y proyectos de innovación con financiamiento externo vigentes.

Los indicadores mencionados se reproducen en diversos sistemas de financiamiento en la Universidad en Chile, tales como el Fondo Basal por Desempeño y una proporción del llamado Aporte Fiscal Directo, así como en el resto de subvenciones. Por ello, como han planteado Sousa et al. (2010), estos indicadores se constituyen a nivel local en los objetivos en torno a los cuales la Universidad se organiza (por sobre lo que las comunidades académicas pudiesen decidir colegiadamente). En tanto la adscripción y organización de la vida universitaria a estos indicadores es condición de financiamiento. Así, bajo la lógica del desempeño y los resultados, se han impuesto normas económicas y políticas vinculadas a la efectividad institucional que privilegian resultados por sobre cómo estos han sido producidos (Sousa et al., 2010).

Tanto el NPM como la EBP presentan una gramática compleja que tensiona el espíritu tradicional de la universidad y la desafía a nuevas éticas y funcionamientos. La reorganización de la universidad sin duda requiere nuevas formas de gestionar el trabajo académico y requiere académicos que puedan cimentar estos cambios mediante su trabajo. Diversos autores señalan que estas reformas deben ser analizadas como un proceso que tiene consecuencias profundas para los académicos (Deem, Hillyard, \& Reed, 2007; Faderdella et al., 2015; Knights \& Clarke, 2013; Thomas \& Davies, 2005).

\section{Dispositivos de gestión y la cuantificación del trabajo académico}

Como se describió en el apartado anterior, un Estado reorganizado bajo la gramática del NPM y EBP imprime nuevas exigencias a la universidad y sus trabajadores. En la última década esto se concreta en la introducción y diseño de inéditas regulaciones 
y dispositivos de gestión del cuerpo académico. Entre los cuales se destaca la masiva incorporación de instrumentos y técnicas de medición para cuantificar aspectos generales y específicos del trabajo universitario. Estos instrumentos han sido denominados por Moore y Robinson (2015) como "dispositivos de cuantificación" y pueden ser definidos (en el contexto laboral) como herramientas para el registro y cálculo sistemático de la producción académica o aspectos puntuales de esta.

El uso de los dispositivos de cuantificación en el ámbito médico es común hace ya algunos años, y ahora comienza también a ser frecuente su uso en los espacios de trabajo. De acuerdo con Moore y Robinson (2015) la propagación de estos dispositivos es permitida por las características actuales del trabajo, así como las transformaciones del trabajo también han sido posibles gracias a estas nuevas tecnologías de gestión. Según Berardi (2009) y Berlant (2011) su aumento se encuentra asociado al aumento de condiciones inestables y flexibles de trabajo, telepresencia, just-in-time y outsourcing.

Según Miller y O'Leary (2007), los dispositivos de cuantificación también podrían ser conceptualizados como instrumentos de mediación, en tanto se trata de instrumentos que transforman procesos complejos y heterogéneos, como es el trabajo cotidiano, en indicadores simples de carácter numérico. Este tipo de instrumentos producen unidades de información que por un lado facilitan la gestión del cuerpo académico, así como la comparación entre los trabajadores. En efecto, no se trata de una consecuencia directa, sino más bien de un ensamble pragmático, donde estos dispositivos (entre otros asuntos), facultan escenarios laborales de constante vigilancia y de intensa competencia. En coherencia con esto, los dispositivos se introducen en la vida laboral como un agente, que funciona mediando como un tercero en la relación empleado/empleado, empleado/ empleador y del empleado consigo mismo. De acuerdo con Moore y Robinson (2015), esto modificaría las coordenadas de fuerzas de poder en el espacio laboral, en tanto hablamos de tecnologías de funcionamiento remoto, invisibles, que facultan la supervisión silenciosa y la competencia ciega en el espacio del trabajo.

Siguiendo a Callon (1998) la mayor fuerza de poder de estos dispositivos radica en su capacidad de modificar el espacio en el que actúan. Callon (1998) los ha identificado como dispositivos con agencia, en tanto si bien tienen la función de representar ciertos aspectos del trabajo, simultáneamente ejercen la función de distinguirlo y relevarlo de otras posibles dimensiones del trabajo. Se trata en definitiva de técnicas analíticas, indicadores y protocolos que se caracterizan por portar demandas, teleologías y/o interpelaciones que intervienen en el espacio de los trabajadores (Muniesa, Millo, \& Callon, 2007).

De acuerdo con Moore y Robinson (2015) la incorporación de estas tecnologías tiene diversas consecuencias sobre la experiencia laboral de los trabajadores. Se remarcan efectos sobre los sujetos trabajadores tales como ansiedad acerca de su rendimiento e incertidumbre respecto de su posible sustitución y estabilidad en la organización (Moore \& Robinson, 2015). También se ha documentado que la cuantificación del trabajo se asocia a la sensación crónica de estar al borde del colapso, la sobre estimulación y 'estrés de la atención' (Berardi, 2009).

\section{Método}

Con el objetivo de dar cuenta del actuar de los dispositivos de gestión y sus efectos sobre el trabajo académico se optó por un método cualitativo de carácter local, específico y contextualizado y por sobre todo, por un interés comprensivo sobre el fenómeno que nos incita a investigar (Denzin \& Lincoln, 2003; Flick, 2004). En coherencia con esto se diseñó un estudio de tipo documental de 95 textos asociados a la gestión de trabajo académico (emitidos entre el año 2005 y 2015). El corpus se constituye por 
documentos heterogéneos, que se dividieron en 3 tipos: Convenios de desempeño (emitidos en coautoría por Universidades y Ministerio de Educación); Bases oficiales para concursos públicos (emitidas por el Ministerio de Educación y/o Comisión Nacional de Investigación Científica y Tecnológica) y documentos internos de gestión del cuerpo académico (emitidos por las instituciones universitarias). La elección de la muestra se realizó bajo el criterio de representatividad del muestreo cualitativo en análisis documental desarrollado por Ibáñez e Iñiguez (1996), donde los textos son representativos en tanto presenten y evidencien los discursos a estudiar y sean a la vez productos discursivos habituales del contexto del estudio (Iñiguez \& Antaki, 1994).

De acuerdo con Prior (2008) los documentos no sólo son producidos por las instituciones, sino que a la vez producen órdenes y acciones particulares en éstas, por ello trabajamos sobre documentos manifiestamente prescriptivos (instructivos, formularios, convenios, resoluciones, protocolos de acción, llamados a concursos académicos).

El análisis se centra justamente en la capacidad de estos documentos de ordenar y regular el trabajo académico. Tomando esto en consideración, se ha seleccionado un método de análisis discursivo pragmático, desde la perspectiva analítica desarrollada por el grupo de Discurso y Retórica de Loughborough (Edwards \& Potter, 1992; Potter, 1998; Wetherell \& Potter, 1998). Este tipo de análisis asume los textos como prácticas discursivas que desarrollan una acción social en su entorno. Entonces su análisis se enfoca en el estudio de estas acciones y el desarrollo de hipótesis sobre los propósitos y consecuencias del texto (Wetherell \& Potter, 1998).

Mediante sucesivas lecturas, bajo la pregunta por la función de los dispositivos de cuantificación en torno al trabajo académico, se codificaron los textos y los códigos se agruparon en cuatro categorías que permiten describir el actuar de los dispositivos: (1) los dispositivos y su nuevo lenguaje para hablar del trabajo académico; (2) los dispositivos y la objetivación de los procesos laborales académicos; (3) los dispositivos, el realce del individuo y omisión del contexto; $y$ (4) los dispositivos como mediadores 'objetivos' de las relaciones laborales académicas.

La nominación de los fragmentos ha sido organizada de la siguiente manera: el nombre en cursiva hace referencia al tipo de documento en que se encuentra la cita, el primer número se refiere al documento y el segundo número, se refiere a la cita respecto de un documento.

\section{Resultados y Discusión}

\section{Los dispositivos y su nuevo lenguaje para hablar del trabajo académico}

La descripción y valoración del trabajo académico puede ser realizada mediante diversos recursos lingüísticos. Cada descripción supone opciones léxicas específicas que no son neutrales, pues tienen efectos en relación a cómo va a ser concebido aquello que se describe, así como aquello que va a ser valorado. Si bien la literatura ha mostrado diversas palabras y conceptos para describir y valorar las tareas académicas, los documentos analizados muestran que los dispositivos de gestión del trabajo académico utilizan un lenguaje específico para referirse a éstos.

A continuación, se muestra un fragmento que permite evidenciar cómo los documentos revisados buscan moldear y orientar mediante sus palabras el ejercicio académico. La cita corresponde a un segmento de las bases del principal concurso nacional de financiamiento a la investigación, donde se explicitan los criterios de elegibilidad de los concursantes:

La productividad del (de la) Investigador(a) Responsable será evaluada por el Grupo de Estudio, considerando un máximo de 10 publicaciones (artículos en revistas científicas, libros y/o capítulos de libros) aceptados, en prensa o publicados a partir 
del año 2009 (Fondo Nacional de Desarrollo Científico y Tecnológico, 2015, p.13).

El texto se refiere a los criterios mediante los cuales se evaluará la 'producción' del investigador: "artículos en revistas científicas, libros y/o capítulos de libro", restringiendo otras posibles producciones académicas (mesas redondas, diálogos académicos, etc.). Adicionalmente, el fragmento establece una temporalidad para la medición de productividad. Esto tiene el efecto de indicar al investigador que su productividad sólo cuenta si es que ha sido realizada en los últimos 5 años, prescribiendo la necesidad de mantenerse produciendo a la vez que señala lo perecedero de dicha producción: todo lo hecho previamente ya no cuenta para su evaluación como investigador.

Un elemento que se destaca de este lenguaje se refiere a que las descripciones y prescripciones se sustentan en formas de habla de carácter imperativo ("será evaluada"), estableciendo categorías lingüísticas de potencia constituyente. Sumado a esto, el carácter oficial de los documentos - en tanto bases para un concurso que condiciona financiamiento para investigar -, favorece su carácter prescriptivo y orientador de acciones de los trabajadores.

Sutilmente, entre palabra y palabra, se erigen los criterios para distinguir las tareas productivas de las 'no productivas' y jerarquizar de esta manera, nuestras acciones. Con ello queda definido qué es lo valorado, qué debe producirse y qué es lo que nos constituye como investigador/a 'productivo/a'.

En consecuencia, si un investigador quiere mostrarse productivo y competir por fondos para llevar a cabo tareas propias de su rol (investigar), debe catalogar e inscribir su trabajo en las categorías que las nuevas regulaciones han dispuesto para ello. De esta forma, su trabajo será leído como productivo. He aquí un ejemplo de cómo los documentos producidos por las nuevas regulaciones, constituyen un código imperativo de inteligibilidad que ordena, organiza y produce determinadas experiencias laborales.

\section{Los dispositivos y la objetivación de los procesos laborales académicos}

Otro aspecto central de los documentos revisados es el énfasis en la medición del trabajo académico y la oferta de diferentes métricas para ello. Cabe destacar cómo los documentos que circulan por nuestros espacios laborales han sido colonizados por cifras, rankings, fórmulas para describir, valorar, organizar y gestionar la producción académica. De esta manera, presenciamos la implementación de tecnologías de cuantificación de lo que antes eran tratados como aspectos inconmensurables y cualitativos del proceso de trabajo.

Progresivamente, mediante estas nuevas tecnologías, todo se va tornando mensurable: la valoración de los estudiantes es medida por encuestas de satisfacción, el impacto de lo que escribimos medido por la citación y/o por la revista en la que publicamos, la cantidad de veces que nos buscan por internet y así un sinnúmero de otras mediciones a las que es sometido nuestro trabajo. Por ejemplo un documento de Políticas de gestión del cuerpo académico señala que:

Para efectos de la calificación bienal de los académicos (...) se establecerán los criterios de productividad y calidad que la Facultad defina como criterios complementarios a aquellos comunes de la Universidad. (...) Para los académicos de categoría ordinaria, los criterios básicos comunes se deberán aplicar a la calidad de la docencia impartida (auto-evaluación del académico, resultados de las encuestas docentes, opiniones y evaluaciones de pares) y la producción en investigación o creación (según estándares internacionales en términos de la cantidad, calidad e impacto de publicaciones o producción de obras). (Pontificia Universidad Católica de Chile, 2008, p.21).

En esta misma línea, la revisión de los documentos relativos a los convenios de desempeño 
firmados entre universidades y el Ministerio de Educación muestra cómo la planificación del trabajo, el cálculo de los costos y los compromisos adquiridos entre instituciones, están mediados por un lenguaje de métricas. Muestra de ello es la siguiente cita:

Las variables para medir el impacto del PMI serán capital humano capacitado, productos desarrollados, protección y transferencia de resultados de investigación al mercado y la sociedad, inserción en redes internacionales de innovación y levantamiento de fondos privados y/o públicos invertidos en innovación (Ministerio de Educación, 2012, p.30).

Sin duda las tecnologías de cuantificación del trabajo académico gradualmente se han ido masificando y sofisticando, logrando que cada vez un mayor número de acciones académicas sean traducidas a un conjunto de indicadores. Moore y Robinson (2015) explican en parte este fenómeno, destacando que las tecnologías de cuantificación ejercen la importante función de transformar una multiplicidad de acciones heterogéneas en indicadores monitoreables, lo cual facilitaría la gestión de la fuerza laboral académica.

Por otra parte, cabe señalar que todas estas mediciones se han instalado bajo la promesa de la objetividad, asunto no menos importante cuando arbitrariamente ésta queda asociada a fenómenos como justicia o probidad en la toma de decisiones de las instituciones. Así queda evidenciado en el siguiente fragmento, donde se explica el mecanismo de jerarquización de los académicos:
(...) se podría llegar a la titularidad por méritos exclusivamente docentes, considerando la calidad de la docencia, la investigación en docencia, la innovación de la enseñanza y otros factores que hoy día pueden ser medidos con bastante objetividad, gracias a los instrumentos que ha elaborado la ciencia pedagógica universitaria (Universidad Austral de Chile, 2007, p.17).
Tal vez, a esta altura de las transformaciones nos parezca natural que el trabajo se organice en torno a números y cantidades o incluso resulte difícil imaginar otras posibles formas de organizar nuestro trabajo.

\section{Los dispositivos, el realce del individuo y omisión del contexto}

Como hemos revisado hasta ahora, los documentos analizados poseen la cualidad de invitarnos constantemente a transformar nuestros procesos de trabajo en sistemas cuantitativos de valor formal. A continuación se muestra un fragmento de un documento oficial Ilamado "Principales Indicadores cienciométricos de la Actividad Científica Chilena". Como se aprecia en el fragmento, un fenómeno complejo como la innovación es reducido y simplificado a fragmentos manejables, tales como "número de trabajos publicados por un dominio dado" o "proporción de los documentos publicados que ha sido citado en patentes respecto del total de la producción del mismo dominio".

\begin{abstract}
Conocimiento innovador - Innovative Knowledge: Número de trabajos publicados por un dominio dado y citada en las patentes. Fuente de datos: PATSTAT (http://www.epo. org). Este indicador es dependiente del tamaño.

Impacto Tecnológico - Technological Impact: Proporción de los documentos publicados que ha sido citados en patentes respecto del total de la producción del mismo dominio. Fuente de datos: PATSTAT (http://www. epo.org). Este indicador es dependiente del tamaño (Comisión Nacional de Investigación Científica y Tecnológica, 2014, p.278).
\end{abstract}

La fragmentación de fenómenos complejos en diferentes artificios medibles es una de las operaciones más comunes de estas tecnologías de la cuantificación, tal como se observó en los documentos analizados. Sin embargo, cabe señalar 
que este procedimiento no se trata de un cálculo que dé cuenta auténtica e íntegramente, de las cualidades de los fenómenos (si se pudiese tal cosa), más bien se trata de un proceso arbitrario de relevar ciertos aspectos de la producción académica por sobre otros.

En términos concretos, aparejado a este procedimiento de fragmentación y reducción encontramos la constante omisión del contexto de la producción académica: por ejemplo condiciones laborales, micro políticas institucionales, dimensiones de género, etcétera. Siguiendo con el fragmento, sabemos que la innovación es un proceso complejo, que tiene que ver con las relaciones laborales que permiten y reconocen procesos creativos, con sistemas sociopolíticos y económicos que valoran " $X$ " como algo innovador. De hecho la innovación entendida como transformación, modificación, creación en un campo específico, al ser transformada en un indicador es reducida a una mera publicación en un dominio específico, y a una citación en patentes. Esto hace que sea la cantidad de veces que esto ocurre, lo que da cuenta del innovar, restringiendo la innovación a una operación administrativa de carácter individualizado, siendo descrita como un atributo del trabajador. Esto también se aprecia en el siguiente fragmento, donde la innovación aparece como una competencia que debe ser desarrollada por los investigadores (Tabla 1).

Tabla 1

Plan de mejoramiento institucional, objetivos generales y específicos, desempeños comprometidos

Objetivo Específico 2

2. Formar competencias asociadas a innovación, protección y transferencia en los equipos de I + D + i de la UC Estrategias por Objetivo Específico 2

2.1 Capacitar a investigadores en in innovación con foco en transferencia de resultados de investigación.

2.2 Capacitar en innovación a estudiantes de doctorado (desarrollo de proyectos aplicados, protección y transferencia de resultados de investigación).

2.3 Capacitar en competencias avanzadas en transferencia a profesionales a cargo de apoyar el proceso de innovación.

Fuente: Ministerio de Educación, 2012, p.31.

Según Kapur (2007), el régimen neoliberal propone una organización del trabajo que idealiza a los trabajadores creativos, realzando las cualidades individuales, desconociendo las dimensiones sociales y contextuales del trabajo. Salmi (2009) ha denominado este fenómeno "voluntarismo mágico", correspondiente a una ideología que elude una realidad de exclusiones, competencias, precariedad de las condiciones laborales al insistir en una capacidad milagrosa de cada individuo. $Y$ la creencia ciega de que los logros y desempeños de los trabajadores corresponden a una capacidad del sujeto y no de la organización (Moore \& Robinson, 2015). De esta forma, frecuentemente bajo estos juegos de palabras se ocultan tecnologías de intensificación e híper responsabilización del trabajador. En efecto, este fragmento muestra no sólo que la innovación se considera una competencia individual que puede ser formada, sino que al apreciar simultáneamente los objetivos específicos, esta competencia es de "transferencia de resultados de investigación". Es decir, la creación y transformación en un campo particular se logra cuando el investigador consigue convencer a otros (especialmente al sector empresarial) de que sus resultados pueden serles de utilidad. En otras palabras la innovación se realiza cuando se despliega una capacidad de transferencia, que como han señalado diversos autores, en términos prácticos es el desarrollo de competencias de venta de un producto a un cliente interesado (Moore et al., 2011). Competencia que queda localizada en el individuo investigador.

\section{Los dispositivos como mediadores 'objetivos' de las relaciones laborales académicas}

La cuantificación se convierte en una forma de mediación entre varios objetos/sujetos/ experiencias y modifica, por tanto, las relaciones 
entre los diferentes elementos que componen los procesos del trabajo académico. Modifica a su vez la relación del académico con sí mismo, situando al vigilante y trabajador dentro del mismo cuerpo, media la relación entre académicos al establecer prioridades y puntajes. Así por ejemplo, cuando se realiza una escritura colaborativa, debemos siempre jerarquizarnos: hay un primer autor que supuestamente trabajó más y un segundo autor que obviamente trabajó menos. Este supuesto se naturaliza e instala en pautas de evaluación e incentivo que usan los sistemas nacionales de evaluación (Fondo Nacional de Desarrollo Científico y Tecnológico es una muestra de ello) y que usan los sistemas institucionales. Hay siempre uno que trabajó más. El siguiente fragmento emerge de un instrumento de evaluación para adjudicar fondos de investigación. Es importante señalar que cada académico debe completar este formulario y su consiguiente puntaje condiciona la obtención de fondos para el desarrollo de su proyecto (Tabla 2).

Tabla 2

Evaluación curriculum. Investigador principal

\begin{tabular}{lccccc}
\hline Publicaciones & \multicolumn{2}{c}{ Puntaje cada uno } & \multicolumn{2}{c}{ Puntaje máximo } & Puntaje asignado por \\
\cline { 2 - 5 } Artículos & Autor & Coautor & Autor & Coautor & evaluador \\
\hline Revistas indexadas & 4 & 2 & 8 & 4 & 4 \\
Revistas no indexadas & 3 & 2 & 6 & Total: puntaje asignado por el evaluador
\end{tabular}

Nota: La tabla 2 corresponde a un fragmento de una tabla original titulada "Evaluación curriculum. Investigador principal" elaborado por la Universidad Metropolitana de Ciencias de la Educación (2016).

Tal como se puede apreciar, no sólo se define productividad en función de publicaciones, generando una jerarquía entre tipos de publicaciones según la indexación de la revista, más que, por ejemplo, la cualidad del escrito. Además de ello se introduce de un modo tácito la noción de que el primer autor es el autor fundamental, asumiendo que quién va en segundo lugar en un artículo ha realizado una contribución menor. Es significativo que ser primer autor es igual a ser autor único, reforzando esta idea. Cabe señalar que los equipos de investigación no pueden definir y/o declarar cuál es el nivel de colaboración de cada uno, como un acto autónomo del equipo autor de un artículo. No, es meramente el orden de autor el que lo establece. Con ello no sólo se acarrean diferencias en puntajes, que además serán públicos, en un ranking académico de la Universidad y/o Facultad, sino que además estas diferencias de puntaje suponen diferencias en las remuneraciones de académicos que trabajan juntos en mismos proyectos, en mismas publicaciones. El dispositivo de gestión desplegado asume que
Como mucho una suma de esfuerzos individuales que son obviamente distintos en nivel, y por ello deben ser recompensados de modo diferenciado. He aquí que se concreta una de las principales premisas gerenciales: el trabajo es individual, debe ser medido e incentivado individualmente, favoreciendo la competencia. Tal como señala Parker (2002), con ello se asume la inexistencia y/o irrelevancia de otras formas de organización del trabajo, y el dispositivo, al actuar, precisamente tiene como efecto desincentivar y eliminar definitivamente de la faz de la Universidad esas formas de trabajo que no calzan con el lenguaje gerencial instalado.

\section{Los dispositivos y su impacto en el trabajo académico}

Los documentos de carácter procedimental analizados despliegan dispositivos de gestión orientados a regular el trabajo académico, estableciendo categorías y métricas que definen y ordenan diferentes aspectos de la labor académica: asignación de valor para las diferentes tareas, 
jerarquías entre académicos, objetivación de diferentes procesos de trabajo, entre otros.

Tal como se señaló, los instrumentos de gestión desarrollan e implementan determinadas opciones respecto al trabajo académico que no son neutrales; se trata más bien de decisiones arbitrarias, representadas por un lenguaje que tiene efectos particulares sobre el trabajo académico. El análisis ofrecido se refiere a documentos que, organizados bajo el patrón de la lógica métrica y la cuantificación, reorganizan el trabajo. Estos discursos asumen lo que Woolgar (1988) y Potter (1998) han denominado la retórica empirista, mecanismo discursivo en el cual los fenómenos son descritos y existen en virtud de acciones que sobrepasan el ámbito de la agencia y el interés humano. Mediante este mecanismo, las actuales formas de organizar el trabajo académico parecen naturales e incluso objetivas. Decisiones arbitrarias, se muestran como formas eficientes y transparentes de gestionar la academia, evadiendo el necesario diálogo detrás de estas nuevas regulaciones. Esto es curioso, considerando que se trata de las regulaciones de un tipo de trabajo que se caracteriza por el debate basado en argumentos y evidencias que deben ser plausibles para la comunidad científica. Por el contrario, se instala un lenguaje objetivo cuya ecuanimidad se basa en cuantificadores de determinados productos y esta métrica se enuncia como garante de dicha objetividad. Sin embargo, los cuantificadores escogidos reducen de un modo drástico lo que se entiende por producción de conocimiento, por innovación o por docencia. Esos cuantificadores operan como representación de lo que es el verdadero trabajo académico en el panel de mando de la gestión de las políticas de producción de conocimiento científico.

Por otro lado, estos dispositivos fomentan una imagen cuantitativa de la productividad humana que, como han mostrado diversas investigaciones, resulta poco adecuada en cuanto a cómo los propios trabajadores académicos consideran su trabajo. De acuerdo con Gill (2009) y Graeber (2011), estos dispositivos de seguimiento provocan la sensación permanente de inadecuación, de falta e incumplimiento, desvalorizando, por otro lado, aquello que los mismos académicos valoran como trabajo académico. La retórica que instala estos dispositivos indica que éstos proporcionarían y construirían datos objetivos sobre las capacidades humanas desplegadas en este trabajo. Sin embargo, sus propios trabajadores no se reconocen en este lenguaje, en sus categorías ni en sus cuantificadores. Tal situación podría intensificar el malestar en los trabajadores académicos por la inadecuación con lo que ellos consideran relevante y por el endeudamiento permanente que lleva a una sensación de insuficiencia constante (Gill, 1995; Graeber, 2011; Moore \& Robinson, 2015).

\section{Consideraciones Finales}

Mediante un análisis pragmático del discurso de documentos oficiales tanto de nivel estatal (Ministerio de Educación y Consejo Nacional para el Desarrollo de la Ciencia y la Tecnología, como institucional (de las propias Universidades), este artículo muestra cómo los instrumentos de gestión promovidos por las actuales políticas de educación superior instalan mandatos y prescripciones que modelan el trabajo académico. En concreto el estudio realizado evidencia que los modos de gestión del trabajo académico y sus dispositivos analizan el trabajo académico mediante acciones tales como: establecer jerarquías entre las múltiples tareas de un académico y entre académicos, mediante criterios que no han sido discutidos por la comunidad profesional; objetivar procesos laborales y asumir consensos en torno a ello, desconociendo disputas y desacuerdos actuales; desconocer el contexto de producción académica, construyendo una imagen del trabajo académico como proceso individual; y finalmente instando a la base del trabajo académico a relaciones laborales individualistas y competitivas.

Los resultados de este trabajo nos llevan a pensar que los nuevos dispositivos de gestión del trabajo académico promovidos por 
organismos internacionales, estatales y asumidos mecánicamente por las instituciones, merecen ser revisados críticamente. En la implementación de estas nuevas regulaciones no sólo se pone en juego el ranking del país en el informe de la OECD, sino también nuestro devenir como comunidad académica y científica, así como nuestro rol en la sociedad. Bajo estas nuevas regulaciones ¿Hacia dónde se orientan nuestras acciones como académicos?, ¿Cómo le otorgamos sentido a nuestro trabajo? ¿Cómo construiremos una comunidad académica? Es necesario profundizar no sólo el análisis de la consistencia de estos 'indicadores' en relación a aquello que suponen medir, sino también en relación a los efectos subjetivos, intersubjetivos e institucionales que están generando en las comunidades académicas. Si la Universidad ha tenido un rol relevante en las sociedades contemporáneas como promotor de progreso y espacio de análisis crítico, locus fundamental del sueño ilustrado de sociedad moderna, los nuevos mecanismos reguladores deben ser analizados seriamente en su capacidad para lograr este sueño.

\section{Colaboradores}

\section{FARDELLA-CISTERNAS y V. SISTO CAMPOS} fueron responsables de la concepción, diseño, análisis e interpretación de los datos. F. JIMÉNEZ VARGAS realizó la revisión y aprobación de la versión final del artículo.

\section{Referencias}

Altbach, P. (2000). The changing academic workplace: Comparative perspectives. Chestnut Hill: Boston College Center for International Higher Education.

Berardi, F. (2009). Precarious rhapsody. New York: Minor Compositions.

Berlant, L. (2011). Cruel optimism. North Carolina: Duke University.

Bernasconi, A. (2015). La educación superior de Chile. Transformación, desarrollo y crisis. Santiago:
Beverungen, A., Dunne, S., \& Sorensen, B. (2008). University, failed: Editorial. Ephemera: Theory and Politics in Organization, 8(3), 232-237.

Blanch-Ribas, J., \& Cantera, L. (2011). La nueva gestión pública de universidades y hospitales: Aplicaciones e implicaciones. In E. Agulló, J. L. Álvaro, A. Garrido, R. Medina, \& I. Schweiger (Eds.), Nuevas formas de organización del trabajo y la empleabilidad. Oviedo: Universidad de Oviedo.

Blanch-Ribas, J., \& Stecher, A. (2009). La empresarización de servicios públicos y sus efectos colaterales: Implicaciones psicosociales de la colonización de universidades y hospitales por la cultura del nuevo capitalismo. In T. Wittke \& P. Melogno (Comps.), Psicología y organización del trabajo: Producción de subjetividad en la organización del trabajo. Montevideo: Psicolibros.

Callon, M. (1998). El proceso de construcción de la sociedad: El estudio de la tecnología como herramienta para el análisis sociológico. In M. Domènech \& F. Triado (Eds.), Sociología simétrica: Ensayos sobre ciencia, tecnología y sociedad. Barcelona: Gedisa.

Castillo, J. (2015). Epistemologías pragmáticas de la vida: Regímenes de valoración y producción de verdad biomédica en el sistema garantías explícitas en salud en Chile. Ponencia presentada en el $30^{\circ}$ Congreso de la Asociación Latinoamericana de Sociología, San José, Costa Rica.

Comisión Nacional de Investigación Científica y Tecnológica. (2014). Principales indicadores cienciométricos de la actividad científica Chilena 2012. Informe 2014: Una mirada a 10 años. Valparaíso: Autor. Recuperado en abril 28, 2017, de http://www.conicyt. cl/informacioncientifica/files/2014/09/IndicadoresCienciom\%C3\%A9tricos-2014.pdf

Deem, R., Hillyard, S., \& Reed, M. (2007). Knowledge, higher education and the new managerialism: The changing management of UK universities. Oxford: Oxford University Press.

Denzin, N., \& Lincoln, Y. (2003). Strategies of qualitative inquiry. Thousand Oaks: Sage.

Edwards, D., \& Potter, J. (1992). Discoursive psychology. London: Sage.

Fardella, C., Sisto, V., \& Jiménez, F. (2015). Nosotros los académicos. Narrativas identitarias y autodefinición en la universidad actual. Universitas Psycológica, 14(5), 15-27.

Flick, U. (2004). Introducción a la investigación cualitativa. Madrid: Morata.

Fondo Nacional de Desarrollo Científico y Tecnológico. (2015). Base oficial a Concurso Fondecyt Iniciación. Recuperado en abril 28, 2017, de http://www.conicyt. 
cl/fondecyt/2015/04/08/concurso-iniciacion-eninvestigacion-2015/

Gill, R. (2009). Breaking the silence: The hidden injuries of neo-liberal academia. In R. Flood \& R. Gill. Secrecy and silence in the research process: Feminist reflections. London: Routledge.

Gill, S. (1995). 'The global panopticon? The neo-liberal state, economic life and democratic surveillance'. Alternatives, 20(1), 1-49.

Gjerding, A., Wilderom, C., Cameron, S., Taylor, A., \& Scheunert, K. (2006). Twenty practices of an entrepreneurial university. Higher Education Management and Policy, 18(3), 83-109.

Graeber, D. (2011). Debt: The first 5000 years. New York: Melville.

Head, B. (2008). Three lenses of evidence-based policy. The Australian Journal of Public Administration, 67(1), 1-11. https://doi.org/10.1111/j.1467-8500.2007.00564.x

Head, B. (2010). Reconsidering evidence-based policy: Key issues and challenges. Policy and Society, 29(2), 77-94. https://doi.org/10.1016/j.polsoc.2010.03.001

Ibáñez, T., \& Iñiguez, L. (1996). Aspectos metodológicos de la psicología social aplicada. In J. Álvaro \& A. Garrido (Eds.), Psicología social aplicada. Madrid: McGraw-Hill.

Ibarra, E. (2002). Capitalismo académico y globalización: La universidad reinventada. Revista de la Educación Superior, 31(122), 147-154.

Iñiguez, L., \& Antaki, C. (1994). El análisis del discurso en Psicología. Boletín de Psicología, 44, 55-75.

Kapur, J. (2007). "New" economy/old labour: Creativity, flatness, and other neoliberal myths'. In C. McKercher \& V. Mosco (Eds.), Knowledge workers in the information society. Lanham: Lexington Books.

Knights, D., \& Clarke, C. (2013). It's a bittersweet symphony, this life: Fragile academic selves and insecure identities at work. Organization Studies, 35(3), 335357. https://doi.org/10.1177/0170840613508396

Miller, P., \& O'Leary, T. (2007). Mediating instruments and making markets: Capital budgeting, science and the economy. Accounting, Organizations and Society, 32(7-8), 701-734. https://doi.org/10.1016/j. aos.2007.02.003

Ministerio de Educación. (2012). Formulario convocatoria de Convenios de Desempeño 2012. Plan de mejoramiento Institucional Definitivo (PMI). Recuperado en abril 28, 2017, de http://mecesup.uc.cl/images/CD/1206/ Documentos/PMI\%20_PUC1206_INES\%201.pdf

Moore, K., Kleinman, D., Hess, D., \& Frickel, S. (2011). Science and neoliberal globalization: A political sociological approach. Theory and Society, 40(5), 505-532.
Moore, P., \& Robinson, A. (2015). The quantified self: What counts in the neoliberal workplace. New Media \& Society, 18(11), 2774-2792. https://doi. org/10.1177/1461444815604328

Muniesa, F., Millo, Y., \& Callon, M. (2007). An introduction to market devices. In M. Callon, Y. Millo, \& F. Muniesa (Eds.), Market devices. Malden: Blackwell.

Organización para la Cooperación y el Desarrollo Económicos. (2009). La educación superior en Chile: Revisión de políticas nacionales de educación. Santiago: Ministerio de Educación.

Parker, M. (2002). Against management: Organization in the age of managerialism. London: Polity Press.

Pontificia Universidad Católica de Chile. (2008). Políticas de gestión del cuerpo académico en la Pontificia Universidad Católica de Chile. Recuperado en abril 28, 2017, de http://www7.uc.cl/dadevra/wseleccion/PDFs/ politicasAcademicas.pdf

Potter, J. (1998). La representación social de la realidad. Discurso, retórica y construcción social. Barcelona: Paidós.

Prior, L. (2008). Repositioning documents in social research. Sociology, 42(5), 821-836. https://doi. org/10.1177/0038038508094564

Ramírez, E. (2013). Future normal: Quantified self-tools at the Apple store quantified self. Retrieved August 12, 2015, from http://quantifiedself.com/2013/01/futurenormal-quantified-self-tools-at-the-apple-store/

Salmi, J. (2009). The challenge of establishing world-class universities. Washington, D.C.: World Bank Publications.

Slaughter, S., \& Leslie, L. (1997). Academic capitalism: Politics, policies and the entrepreneurial university. Baltimore: The Johns Hopkins University Press.

Slaughter, S., \& Rhoades, G. (2004). Academic capitalism in the new economy: Markets, state and higher education. Baltimore: The Johns Hopkins University Press.

Sousa, C. A. A., Nijs, W. F., \& Hendriks, P. H. J. (2010). Secrets of the beehive: Performance management in university research organizations. Human Relations, 63(9), 14391460. https://doi.org/10.1177/0018726709357083

Svensson, P., Spoelstra, S., Pedersen, M., \& Schreven, S. (2010). The excellent institution: Editorial. Ephemera: Theory and Politics in Organization, 10(1), 1-6.

Thomas, R., \& Davies, A. (2005). Theorizing the micropolitics of resistance: New public management and managerial identities in the UK public services. Organization Studies, 26(5), 683-706. https://doi. org/10.1177/0170840605051821

Universidad Austral de Chile. (2007). Fundamentos conceptuales de la carrera académica para la Universidad Austral de Chile. Documento de trabajo dirigido al 
Consejo Académico. Recuperado en abril 28, 2017, de http://intranet.uach.cl/organizacion/rectoria/pdf/ fundamentos_conceptuales_carrera_academica.pdf

Universidad Metropolitana de Ciencias de la Educación. (2016). Concurso interno de Investigación, pauta evaluación proyectos APEX. Primer semestre 2016. Recuperado en abril 28, 2017, de http://www. umce.cl/joomlatools-files/docman-files/universidad/ direcciones/d_investigacion/concurso_diumce/ concurso-2016/pauta-evaluacion-apex-2016.pdf

Wetherell, M., \& Potter, J. (1998). El análisis del discurso y la identificación de los repertorios interpretativos.
In A. Gordo \& J. Linaza (Eds.), Psicología, discurso y poder: Metodologías cualitativas, perspectivas críticas. Madrid: Visor.

Woolgar, S. (1988). Science: The very idea. London: Routledge.

Recibido: Abril 6, 2015

Versión final: Septiembre 16, 2016

Aprobado: Octubre 19, 2016 